\title{
LA DINÁMICA SOCIAL EN LA DEFINICIÓN DEL ESPACIO RURAL
}

\section{THE SOCIAL DYNAMIC IN THE DEFINITION OF THE RURAL SPACE}

\author{
Wilson González 1 \\ ${ }^{1}$ Ingeniero Agrónomo, M.Sc. Docente. Universidad Pedagógica y Tecnológica de Colombia. Facultad de Ciencias Agropecua- \\ rias. Ingeniería Agronómica. Tunja, Boyacá, Colombia.wilson.gonzalez@uptc.edu.co
}

Rev. U.D.C.A Act. \& Div. Cient. 14(1): 93 - 99, 2011

\section{RESUMEN}

La dinámica del espacio rural obliga a una redefinición del acervo conceptual propio para su interpretación. En este sentido, el concepto de territorio trasciende los tradicionales encuadres políticos, para atender las construcciones sociales vinculadas a dicho espacio. El presente documento resultó de la construcción teórica para el desarrollo del proyecto de investigación titulado "La nueva ruralidad en el quehacer académico de los estudiantes de Ingeniería Agronómica: cuna necesidad en el actual modelo educativo?, realizado en la Universidad Pedagógica y Tecnológica de Colombia UPTC, con sede en el municipio de Tunja, Colombia". El razonamiento deductivo inició con el análisis de los principios del enfoque territorial para concentrarse en la definición del espacio rural, desde los vínculos de los actores que lo acompañan. El razonamiento destaca la importancia de los vínculos sociales en la definición del territorio y propone la necesidad de la incorporación de la dinámica socio temporal, para la interpretación del espacio rural.

Palabras clave: Espacio rural, territorio, contexto, dinámica social, interacción social, nueva ruralidad.

\section{SUMMARY}

The dynamics of the rural space, forces a new definition of the peculiar conceptual estate for its interpretation. In this sense, the concept of territory extends beyond the traditional political limits in order to meet their social constructions. This document results from the theory construction for the development of the research project: "The new rurality in the academic task of agricultural engineering students: a necessity in the current educational model?" carried out at the Pedagogical and Technological University of Colombia, UPTC, located at Tunja, Colombia. Deductive reasoning started with the analysis of the origin of the territorial approach to concentrate in the definition of the rural space, beginning with the participating actors association. The reasoning emphasizes the importance of the social links within the definition of territory and proposed the necessity of the incorporation of the social temporal dynamic for the interpretation of the rural space.

Key words: Rural space, territory, context, social dynamic, social interaction, new rurality.

\section{Consideraciones iniciales}

La existencia de múltiples condicionantes en la "esfera rural latinoamericana" ha generado un espacio particular, cuya especificidad y dinámica lo distancia de las interpretaciones, "convencionalmente aceptadas", por la sociología rural referidas al territorio, donde las distinciones entre lo rural y lo urbano constituyen su andamiaje conceptual (Hillyard, 2007). En la actualidad, los esfuerzos conceptuales por interpretar la dinámica del espacio rural suponen una atenta revisión de las relaciones ocurrentes, entre la población vinculada a dicho ámbito y su entorno.

La necesidad de rebasar el enfoque sectorial de lo rural y lo urbano ha permitido soportar propuestas fundamentadas en un análisis detenido de lo que involucra la relación entre estos dos ámbitos y, consecuentemente, conllevado al estudio de sus distintos procesos de articulación (González E Álvarez, 2008).

En este sentido, han surgido enfoques teóricos, tales como el de la multifuncionalidad rural y la nueva ruralidad, como 
respuesta a una interpretación de la dinámica del espacio rural en América Latina y la Unión Europea, respectivamente, y cuyo radio de acción constituye el análisis de la dinámica rural, en un contexto privilegiado por la maraña de relaciones, producto de su inevitable inserción al escenario globalizante (Llambi, 2004).

Es importante precisar que los distintos escenarios admiten la especificidad del enfoque en función de las características inherentes a cada contexto. En este sentido, si bien es cierto, la Multi - Funcionalidad de la Agricultura (MFA) y la Nueva Ruralidad coinciden en la necesidad de trabajar los fundamentos, que permitan redefinir las políticas rurales: la primera, da relevancia al estudio del mercado y su capacidad para regular la agricultura, mientras que la Nueva Ruralidad intenta explicar el proceso de correlación entre las actividades vinculadas al espacio rural y su inserción en la liberalización comercial (Bonnal et al. 2004).

El presente artículo indaga sobre los condicionantes que determinan la redefinición del espacio rural, así como sus implicaciones para la ingeniería agronómica. La reflexión soportó la etapa descriptiva del proyecto titulado "La nueva ruralidad en el que hacer académico de los estudiantes de Ingeniería Agronómica: cंuna necesidad en el actual modelo educativo?, realizado en la Universidad Pedagógica y Tecnológica de Colombia UPTC, con sede en el municipio de Tunja, Colombia. Para su elaboración, se siguió el método deductivo de investigación, en el cual, a partir de los fundamentos del enfoque territorial con relación a la definición del espacio rural, se concentró el análisis en la explicación de las relaciones entre lo rural y lo urbano, para finalizar con el capital social, como fundamento de dichas correspondencias. El bagaje teórico para la reflexión incluyó las apreciaciones conceptuales propias de la Nueva Ruralidad, haciendo énfasis en las concepciones ideológicas de autores latinoamericanos.

\section{¿Un espacio rural?}

La capacidad de interacción entre el ser humano y su entorno determina, no solo su quehacer diario sino, muy especialmente, moldea su espacio de acción. Esta consideración toma singular importancia a la luz del proceso de definición del espacio rural.

De acuerdo con Rojas (2008), el acervo cultural estipula la diferenciación particular de los diversos lugares, en función de las interrelaciones existentes en dichos espacios, quienes, a su vez, moldean las representaciones culturales que los caracterizan.

Las ideas expuestas permiten inferir que la visión de territorio escapa los "tradicionales" encuadres políticos, que delimitan las regiones y se concentra en las especificidades socioculturales, características de una población y que resultan de su acervo colectivo.

Schejtman E. Berdegué (2004) indican que el territorio no es un espacio físico, objetivamente existente, sino una construcción social, es decir, un conjunto de relaciones sociales que dan origen y, a la vez, expresan una identidad y un sentido de propósito compartido por múltiples agentes públicos y privados.

La peculiaridad del territorio, como función del quehacer del colectivo vinculante, se traduce en el trasegar de los distintos actores por las dimensiones resultantes de su actividad, como miembros de una sociedad. La figura 1 ayuda a comprender este fenómeno. Nótese que los factores internos (endógenos) y externos influyen en el quehacer de los actores en las distintas dimensiones (social, política, económica, ambiental).

Se puede deducir que el rasgo territorial es determinado, tanto por la confluencia interna, propia de la interacción poblacional, como por la concurrencia de los factores "externos", que intervienen en un espacio.

Con este corolario, se debe admitir que el análisis del espacio rural parte por reconocer, tanto las relaciones endógenas como las externas, que suceden en los distintos ámbitos propios del quehacer de sus habitantes. Desde esta perspectiva, una explicación de cambios en lo rural obedece a un viraje en la naturaleza y en la dinámica de las interacciones de sus habitantes.

\section{El enfoque territorial}

Las líneas anteriores permitirían sugerir que un espacio de confluencia de relaciones entre los participantes en los distintos ámbitos puede soportar los pilares para la construcción de una concepción de territorio. Esta última acepción ha sido utilizada por enfoques, como el Desarrollo Rural territorial, como sustento fundamental de su estrategia de desarrollo rural. En este sentido, el territorio implica una cimentación social, que se distancia de los límites políticos o económicos. Así, los procesos de desarrollo rural se asientan en un espacio particular, definido por las relaciones establecidas entre los agentes que lo conforman y su entorno inmediato.

El bagaje teórico contenido en los postulados del Desarrollo Económico Local y del capital social direccionan el enfoque territorial rural y los principios que lo fundamentan: innovación, relación rural - urbana e institucionalidad y capital social (Ruiz E Delgado, 2008).

Es importante precisar que en este estado del documento solo se ha discutido sobre la noción de territorio utilizada 

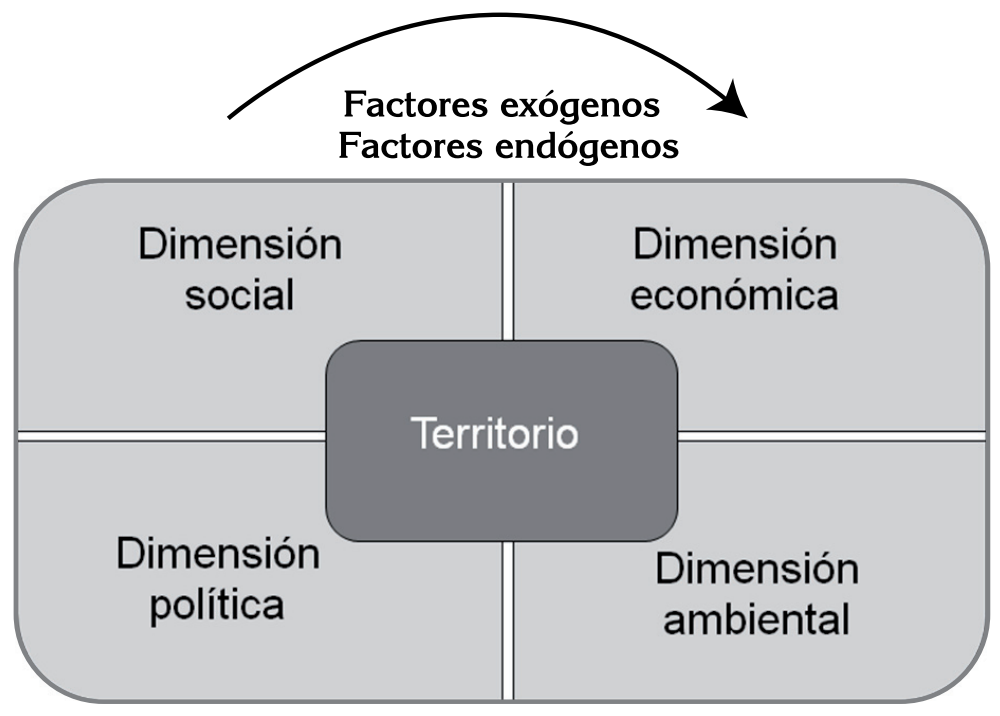

Figura 1. Territorio como construcción social.

en el enfoque del Desarrollo Territorial Rural. Esta situación, no omite que dicha definición es inherente a la estrategia de desarrollo, que promueve "un proceso de transformación productiva e institucional en un espacio rural determinado, cuyo fin es reducir la pobreza rural" (Schejtman E Berdegué, 2004).

De la misma manera, dicho enfoque demanda la denominada "governancia", entendida como "un proceso abierto de coordinación económica, social y política entre una multiplicidad de actores e intereses (públicos y privados) que intervienen en territorios rurales" (Gorenstein et al. 2007).

El enfoque territorial intenta aproximarse al espacio rural con el estudio de la dinámica resultante de las relaciones inherentes a su entorno. Consecuentemente, para dicho acercamiento, el vínculo rural - urbano adquiere una singular importancia, si se tiene en cuenta, que es, precisamente, el que configura el territorio.

Antes de avanzar, conviene puntualizar que el estudio de las articulaciones inherentes a los ámbitos: rural - urbano debe tener en cuenta que las relaciones que allí suceden son fruto de la singularidad contenida en estos espacios. La figura 2 permite apreciar que las decisiones tomadas en el quehacer cotidiano de la población (D1 y D2) surgen como consecuencia de las relaciones e interacciones de los espacios rural y urbano y sus vínculos con el escenario globalizante.

El esquema propuesto permite inferir que un territorio se define en función de los lazos que generan los actores participantes en un determinado espacio. De esta manera, circunscribir geográficamente el espacio existente en un determinado territorio solo tiene sentido, si dicho espacio posee el arraigo de su conjunto poblacional.

López et al. (2005) definen el territorio como "un espacio geográfico sobre el cual los grupos humanos (individuos, colectivo de personas, empresas o instituciones) ejercen un dominio y han creado con este vínculos de pertenencia, identidad y apropiación, cargándolo de un significado particular". El estudio del territorio exige la integración y la comprensión de sus valoraciones multidimensionales que lo caracterizan: políticas, ambientales, culturales, económicas (Pérez, 2004).

Si se tienen en cuenta estas consideraciones es pertinente indagar por los protagonistas de las relaciones que determinan la configuración del territorio.

Las relaciones sociales que confluyen en un territorio, se explican por los vínculos establecidos entre los protagonistas de un espacio y la sincronía organizacional del colectivo inherente a dicho espacio, es decir, en un territorio existen distintos "niveles" de correspondencia social.

La perspectiva teórica del denominado capital social, entendido este último, como "el contenido de ciertas relaciones y estructuras sociales, es decir, las actitudes de confianza que se dan en combinación con conductas de reciprocidad y cooperación" (Durston, 2002), se convierte en una importante herramienta al considerar la dinámica social en función de la especificidad de las relaciones ocurrentes en un espacio. 


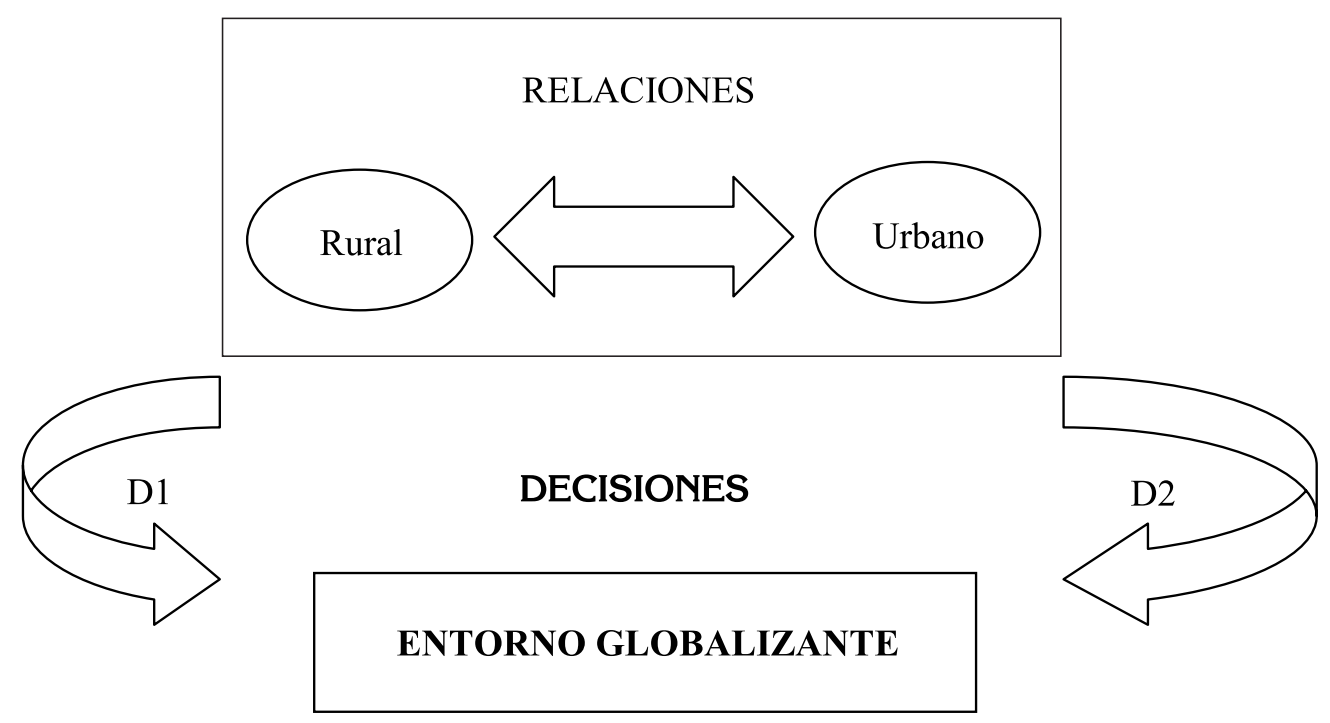

Figura 2. Decisiones en los ámbitos rural y urbano. Fuente: Adaptado de González \& Álvarez (2008).

Siguiendo a Woolcok (2000), citado por Pórtela E Neira (2002), el capital social "simplemente es el reflejo de la realidad de los vínculos sociales en los cuales nos apoyamos en las situaciones de incertidumbre, cuando queremos realizar nuestras aspiraciones o cuando queremos lograr objetivos que no conseguiríamos por nosotros mismos".

Si bien es cierto, la dinámica social, explicada con la concurrencia de las distintas relaciones, se encuentra mediada por mecanismos de autonomía, en los que las acciones colectivas y las directrices normativas propias de sus estructuras organizacionales, tienen una alta injerencia; también lo es, que los niveles de sinergia, generados por el capital social, modifican su dinámica. En esta situación, el ser humano tiene una especial relevancia en función de su disposición a aceptar o las invitaciones del entorno.

El análisis supone que el capital humano, centrado en las habilidades y en las competencias de los individuos (Pórtela \& Neira, 2002), define y particulariza las relaciones que se dan en el colectivo (capital social).

Granada (2001) señala la importancia del espacio personal, definido -desde la psicología social- como el entorno que rodea un individuo en el afirmamiento de las distancias personales y los procesos de interacción social.

La descripción de territorio no pretende omitir la acepción de espacio. La espacialidad y la temporalidad son elementos inherentes a la correspondencia social y determinan la dinámica del territorio. Singularizar la dinámica territorial es analizar por la espacialidad y la temporalidad de sus relaciones.
Suponer, entonces, que las relaciones sociales admiten una estrecha correlación con los procesos sociales, cuya temporalidad y espacialidad definen un territorio, parecería ser el hilo conductor del presente ejercicio.

Si se admite que la dinámica del entorno motiva también un acomodamiento de sus actores vinculantes, en función de particulares estrategias de adaptación, se puede comprender, entonces, que los procesos sociales corresponden a dinámicas evolutivas.

Desde una óptica económica, el paradigma evolutivo, denominado "sociomática", intenta modelar los fenómenos socio-económicos en función de su adaptación al entorno (Castañeda, 2009).

De acuerdo con lo anterior, las características del capital social, fundamento de la correspondencia social, explican la dinámica de los procesos sociales, que se configuran en un tiempo y en un espacio determinado.

\section{Diferentes ruralidades $\mathrm{y}$ distintos procesos de desarrollo}

Hemos llegado a saber que todo individuo vive, de una generación a otra, en una sociedad, que vive una biografía y que la vive dentro de una sucesión histórica (Mills, 1995).

Cada momento y lugar adquieren una particularidad en función de la dinámica, resultante de la articulación de las actuaciones de la población vinculante. Si bien es cierto, los 
objetos naturales (dones del espacio geográfico) provienen directamente de la naturaleza, las prácticas humanas relacionadas con ellos las incorporan y las apropian teniendo en cuenta las intencionalidades y las acciones sociales (Montañez, citado por López et al. 2005).

Al entender que la movilidad de las relaciones es determinante para explicar la concepción de territorio, se puede afirmar que las interpretaciones del espacio rural se acercan a su objeto de estudio, en función de su capacidad, para comprender la dinámica socio temporal existente. En este sentido, distintas ruralidades explican diferentes territorios.

A la luz del proceso globalizante y la "inevitable" incorporación de los mercados al libre comercio, con la consecuente "homogenización" de las condiciones para la comercialización, la concepción de territorio adquiere una singular importancia, en la medida en que distintos territorios requieren, para su comprensión, la exploración de las relaciones particulares al mismo.

Esta última afirmación se refiere, en particular, a la disponibilidad de información por parte de los actores en los flujos de mercadeo. La particularidad de los nichos, así como la renta diferencial y consecuentemente, la especificidad de perfiles de consumidores, no es desconocida.

La competitividad, como referente del proceso globalizante y definida como "la capacidad de una organización económica (empresa, eslabón o cadena productiva) para mantener, conquistar o ampliar un participación en el mercado, incluido el interno, de una manera rentable que permita su crecimiento y sea sostenible en el largo plazo" (Espinal E Martínez, 2005) y la apertura mercantil, suponen también una adaptabilidad de las regiones en función de su aptitud y, consecuentemente, de sus ventajas competitivas.

Un análisis del concepto, desde la óptica territorial, obliga a responder ciertos cuestionamientos:

La rentabilidad, como elemento fundamental de la competitividad cंes diferencial?, y si lo es ċcómo concebir unos indicadores homogéneos para cuantificar el crecimiento y la sostenibilidad?

Los territorios como espacios reveladores de la dinámica social presentan diversas características en función de su capital social. Los vínculos de confianza, de reciprocidad y de cooperación operan en un contexto determinado por los recursos naturales y las elaboraciones sociales, propias de un espacio. En este sentido, los procesos de decisión de los actores, así como las racionalidades contenidas en ellos son complejos y no admiten una uniformidad conceptual.

Si bien es cierto, en el contexto rural latinoamericano, existe una "coincidencia" en la tipificación conceptual de los esquemas productivos, a saber: agricultura tradicional, pequeña agricultura empresarial y agricultura propiamente empresarial, diferenciables en razón de sus distintas condiciones económicas y de inserción al mercado (Espinal $\mathcal{E}$ Martínez, 2005) (Cuadro 1), que conducen a interpretaciones fraccionadas y a políticas diferenciadas, que también lo es en la práctica, los mecanismos de interacción poblacional, que resultan en la convivencia de innumerables estrategias de supervivencia, que no es posible encasillarlas a una tipología particular. Bajo esta óptica, no solo las economías de escala explican las diferencias en la rentabilidad del sistema

\section{Cuadro 1. Esquemas productivos en Latinoamérica.}

\begin{tabular}{|c|l|}
\hline \multicolumn{1}{|c|}{ Tipo } & \multicolumn{1}{c|}{ Característica } \\
\hline Agricultura tradicional & $\begin{array}{l}\text { Estructura familiar } \\
\text { Baja orientación al mercado } \\
\text { Autoconsumo } \\
\text { Bajo valor agregado en la producción }\end{array}$ \\
\hline Pequeña agricultura empresarial & $\begin{array}{l}\text { Tiende al monocultivo } \\
\text { Orientación al mercado } \\
\text { Precaria organización empresarial }\end{array}$ \\
\hline Agricultura empresarial & $\begin{array}{l}\text { Estructura empresarial compleja y formal } \\
\text { Alto desarrollo tecnológico e inversión } \\
\text { Economías de escala }\end{array}$ \\
\hline
\end{tabular}

Adaptado de Echeverri (2002). 
Los sistemas productivos resultan -en la práctica- del arreglo de distintas estrategias de supervivencia que realizan los individuos en un territorio determinado. De acuerdo con González (2009), una adecuada aproximación al espacio rural debe contener un análisis que trascienda los conceptos meramente económicos y se concentre en el estudio de las interacciones poblacionales en un espacio y, consecuentemente, abrigue los distintos criterios de decisión de sus actores participantes. Igualmente, "las relaciones funcionales entre los espacios urbano-rurales" son uno de los estudios fundamentales para emprender en el proceso de la liberalización económica, sin detrimento de los contextos locales (Pérez, 2004).

En particular, interpretaciones dadas a sectores, tales como la economía campesina, no solo exigen entender la racionalidad de las acciones propias de sus participantes, sino la moralidad de las mismas.

El criterio de decisión de un campesino resulta del cotejo de innumerables variables que buscan optimizar su renta agrícola familiar. En este sentido, su supervivencia se explica -entre otras razones- en términos de la maximización del valor de la producción, incluyendo el autoconsumo o de la minimización de los factores externos a la producción. Las estrategias citadas requieren de un marcado acento de mutualismo social.

González (2009) explica la permanencia de la cadena agroalimentaria de la papa en Colombia, desde esta perspectiva. En este ejercicio, se encontró quela maximización de la renta agrícola familiar se puede relacionar con una disminución del punto de equilibrio, calculado en función de la estructura de costos; dicha situación explica la continuidad de este sistema, aun ante drásticas disminuciones del precio de venta del tubérculo.

\section{Cuestión final}

Los aportes anteriormente expuestos permiten sugerir que la interpretación fraccionada de la realidad obedece a razones antropocéntricas que intentan explicar el abordaje de líneas de acción a un determinado componente en el quehacer comunitario; no obstante, la dinámica socio-temporal de los territorios, entendidos como una construcción social, obliga a cuestionar tal segmentación.

Si bien es cierto, caracterizar una población supone un acercamiento a su problemática, también lo es, que diferenciarla en función de usanzas conceptuales, limita dicha aproximación. En particular, interpretar el espacio rural exige una observación permanente de su dinámica $y$, consecuentemente, un desarraigo de los paradigmas conceptuales para su definición.

En consecuencia, la redefinición del espacio rural obliga también a repensar sobre las estrategias de intervención. De acuerdo con Rodríguez (2004), la evolución de los paradigmas exige a una co-evolución de las tendencias y modalidades de intervención.

En particular, para la Ingeniería Agronómica, un viraje en su espacio preferente de acción, precisa un cuestionamiento de los pilares, sobre los cuales, descansa su enseñanza. De esta manera, es necesario trascender los enfoques reduccionistas, como las apuestas técnicas a soluciones agrícolas para entender los procesos de cambio sucedidos en una categoría territorial, denominada ruralidad, no ligada a alguna actividad específica (Llambi, 2004). Lo anterior, compromete la inclusión de estudios transdisciplinares, en los que el dialogo entre las "ciencias exactas" y las "ciencias sociales" sea el fundamento para comprender la valoración multidimensional de territorio.

\section{BIBLIOGRAFÍA}

1. BONNAL, P.; BOSC, P.; DÍAZ, J.; LOSCH, B. 2004. Multifuncionalidad de la agricultura y nueva ruralidad. En: Pérez, E.; Farah, M. Compiladoras. Desarrollo Rural y Nueva Ruralidad en América Latina y la Unión Europea. Pontificia Universidad Javeriana. Ed. Javegraf. (Bogotá). p.19-41.

2. CASTAÑEDA, G. 2009. "Sociomatica": El estudio de los sistemas adaptables complejos en el entorno socioeconómico. El Trimestre Económico. (México). 76(1):5-60.

3. DURSTON, J. 2002. El capital social. En: CEPAL. El capital social campesino en la gestión del desarrollo rural. (Santiago de Chile). p.15-42.

4. ECHEVERRI, R. 2002. El territorio y la economía de los Recursos naturales. En: IICA. Nueva Ruralidad Visión del territorio en América Latina y el Caribe. (Costa Rica). p.83-101.

5. ESPINAL, C.; MARTÍNEZ, H. 2005. La competitividad de las cadenas agroproductivas en Colombia. Ministerio de Agricultura y Desarrollo Rural. (Colombia). 940p.

6. GONZÁLEZ, W. 2009. Educación y entorno en la Ingeniería Agronómica: un encuentro entre la academia y su contexto. Universidad Pedagógica y Tecnológica de Colombia. (Colombia). 53p. 
7. GONZÁLEZ, W.; ÁLVAREZ, J. 2008. La nueva ruralidad en el quehacer académico de la ingeniería agronómica. Revista Ciencia y Agricultura. (Colombia). 6(1):35-40.

8. GORENSTEIN, S.; NAPAL, M.; OLEA, M. 2007. Territorios agrarios y realidades rururbanas. Reflexiones sobre el desarrollo rural a partir del caso pampeano bonaerense. Revista Eure. (Chile). 33(100):91-113.

9. GRANADA, H. 2001. El ambiente social. Revista Investigación y Desarrollo. (Colombia). 9(1):388-407.

10. HILLYARD, S. 2007. Sociology of Rural Life. Berg Publishers. (USA). 186p.

11. LLAMBI, L. 2004. Nueva ruralidad, multifuncionalidad de los espacios rurales y desarrollo local Endógeno. En: Pérez, E.; Farah, M. compiladoras. Desarrollo Rural y Nueva Ruralidad en América Latina y la Unión Europea. Pontificia Universidad Javeriana. Ed. Javegraf. (Bogotá). p.91-100.

12. LLAMBI, L. 2004. La enseñanza del desarrollo rural con enfoque territorial en América Latina. En: Pérez, E. compiladora. La enseñanza del Desarrollo Rural: enfoques y perspectivas. Pontificia Universidad Javeriana. Ed. Javegraf. (Bogotá). p.163-179.

13. LÓPEZ, J.; DELGADO, D.; VINASCO, L. 2005. La interfase urbano rural como territorio y espacio para la sostenibilidad ambiental. Revista Ingenierías. (Colombia). 4(7):29-41.

14. MILLS, W. 1995. La imaginación sociológica. Fondo de Cultura Económica. (México). 236p.
15. PÉREZ, M. 2004. Valoraciones conceptuales y énfasis de la planificación en la formación de gestores. En: Pérez, E. compiladora. La enseñanza del Desarrollo Rural: enfoques y perspectivas. Pontificia Universidad Javeriana. Ed. Javegraf. (Bogotá). p.223-252.

16. PÓRTELA, M.; NEIRA, I. 2002. Capital social: concepto y estudio econométrico sobre el capital social en España. Revista Estudios económicos de desarrollo internacional. (España). 2(2):25-52.

17. RODRÍGUEZ, R. 2004. El espacio rural como paradigma teórico. En: Pérez, E.; Rodríguez, R. coord. Espacios y desarrollos Rurales: una visión múltiple desde Europa y Latinoamérica. Trea. (España). p.9-21.

18. ROJAS, J. 2008. La agenda territorial del desarrollo rural en América Latina. Revista Observatorio de la Economía Latinoamericana. (96). Disponible desde internet en: http://www. eumed.net/cursecon/ecolat/ la/ (con acceso 02/02/10).

19. RUIZ, N.; DELGADO, J. 2008. Territorio y nuevas ruralidades: un recorrido teórico sobre las transformaciones de la relación: campo-ciudad. Revista Eure. (Chile). 34(102):77-95.

20. SCHEJTMAN, A.; BERDEGUÉ, J.A. 2004. Desarrollo territorial rural. Documento FIDA-BID. (Chile). 53p.

Recibido: Julio 28 de 2010

Aceptado: Marzo 24 de 2011 